\title{
Vehicle Motion Control Issues Using Micro Electric Vehicle "NOVEL"
}

\author{
Pongsathorn Raksincharoensak*, Motoki Shino** and Masao Nagai***
}

\begin{abstract}
This article describes the scope of vehicle dynamics control application on micro-scale electric vehicle for enhancing its active safety. The use of in-wheel-motor control in various control objectives as active safety devices are stated in the paper. In the first half of the paper, the control systems for enhancing vehicle dynamics are described. The active front steering by using steer-by-wire mechanism for improving vehicle handling and stability is mainly presented. The latter half of the paper presents the control system designed for driving assist system. The lane keeping control with torque distribution strategy is described. The theoretical controller design and experimental validation are shown in the paper.
\end{abstract}

Keywords: Battery Electric Vehicles, Personal Mobility, Wheel Motor, Steer-by-Wire, Vehicle Dynamics

\section{INTRODUCTION}

Nowadays, from the viewpoint of environmental concerns and energy conservation, advanced researches as well as development efforts in the field of low-emission vehicles have been extensively conducted. Among several types of low-emission vehicles, pure electric vehicles (EV) are expected to play a key role in public and private transportation systems in the near future. Especially, it is expected that micro-scale electric vehicle will act as a new mobility between pedestrians and conventional automobiles. However, passive safety system of small-size vehicle cannot be expected to protect passenger when crash accident occurs, therefore the active safety development for preventing accidents is necessary. Until now, study on active safety of such vehicle is relatively sparse when compared to conventional engine vehicle, so it is essential to secure drive safety of micro-scale electric vehicle[1-2]. Electric actuators which precisely control torque and angle have been widely used in automobile electronic chassis control system. This paper mainly describes the scope of utilizing electric motor for controlling vehicle dynamics. For the design of electric vehicle drive system, the sophisticated configuration called "In-Wheel-Motor" have been developed by integrating the motor into each drive wheel so that those motors can be controlled independently of each

\footnotetext{
* Tokyo University of Agriculture and Technology, 2-24-16 Naka-cho Koganei Tokyo 184-8588, e-mail:pong@cc.tuat.ac.jp

** The University of Tokyo,

7-3-1 Hongo, Bunkyo-ku, Tokyo 113-8656 e-mail: motoki@sl.t.u-tokyo.ac.jp

*** Tokyo University of Agriculture and Technology,

2-24-16 Naka-cho Koganei Tokyo 184-8588,

e-mail:nagai@cc.tuat.ac.jp
}

other. Therefore, it is expected that these structural merits of electric vehicles can effectively expand the degree-of-freedom in the design of active safety technology. For example, in critical driving situations, the vehicle motion can be stabilized by yaw moment generated as a result of the difference in tire traction (or braking) forces between the right and left which is generally called "Direct Yaw-moment Control"(DYC).

Besides DYC system, as a part of the paper, the active front steering system utilizing electric motor is also studied. The configuration of steer-by-wire which the front wheel and the steering wheel do not connect each other has potential to control vehicle actively without the mutual interference between the driver and the control system.

The first half of the paper discusses about the steer-by-wire system which is equipped for the other micro electric vehicle "NOVEL-II". It consists of two electric AC servo motors which are used for the control of front steering angle and steering reaction torque. The controller design and experimental validation are described. In the latter half of the paper, a chassis control system which utilizes DYC as lane keeping assistance system for driver steering workload reduction. The electric vehicle "NOVEL-I" equipped with two wheel-motors is used for experiment study. The functionality of lane keeping control with DYC are demonstrated and its effectiveness on lane keeping are verified.

\section{EXPERIMENTAL VEHICLES}

The micro electric vehicle "NOVEL-I" has two in-wheel-motors equipped at the rear axle which can distribute the driving torque in transverse direction. In addition, the vehicle-mounted CCD camera is used to 
recognize the lane marker and measure the preview lateral deviation in front of vehicle, together with PC for image processing. To control each in-wheel-motor independently with drive-by-wire, the vehicle is equipped with DSP-embedded PC. For sensing the driver operation and vehicle motion, a number of sensors are equipped on the vehicle, such as steering wheel angle sensor, gyro sensor for yaw rate measurement, accelerometer for acceleration measurement, and rotary encoder for wheel velocity measurement.

The other micro electric vehicle "NOVEL-II" is equipped with the steer-by-wire (SBW) system consisting of reaction torque motor for adjusting steering feeling and front-tire angle motor for steering the front tires. SBW system is practically realized by two steering motors. As a fail-safe mechanism, this system is equipped with an electric magnetic clutch between column shaft and intermediate shaft that connects them together as conventional mechanical linkage. The experiment vehicle is equipped with vehicle motion sensors and steering motors in which their signals are processed via DSP system. The measurement values are used to calculate the command angle of steering motor, and steering reaction torque.

\section{STEER-BY-WIRE CONTROL}

For enhancing handling and stability of vehicle, the yaw rate is controlled to trace the desired value by using SBW system. With the application of model matching control technique, the control system consists of a feedforward compensator with respect to the steering wheel angle, and a feedback compensator by using theory of disturbance observer. The proposed SBW control system on NOVEL-II can be expressed as the block diagram as shown in Fig. 2 .

As a previous work, the SBW tracing the yaw rate response of zero-side-slip 4WS was proposed to achieve the theoretical value of steering gear ratio and derivative steering gain. The active steering angle can be expressed as the following expression.

$$
\delta_{f f}(s)=\frac{1}{N}\left(1+\frac{\tau_{\gamma}-\tau_{\gamma d}}{\tau_{\gamma d} s+1} s\right) \delta_{s w}(s)
$$

where, each parameter is determined as follows:

Gear ratio : $N=\frac{\left(1+\frac{m l_{r}}{2 l l_{f} C_{f}} V^{2}\right)}{\left(1+\frac{m}{2 l^{2}} \frac{C_{r} l_{r}-C_{f} l_{f}}{C_{f} C_{r}} V^{2}\right)} \frac{l_{f}}{l} n$

Time response : $\tau_{\gamma d}=\frac{I V}{2 l l_{f} C_{f}+m l_{r} V^{2}}$

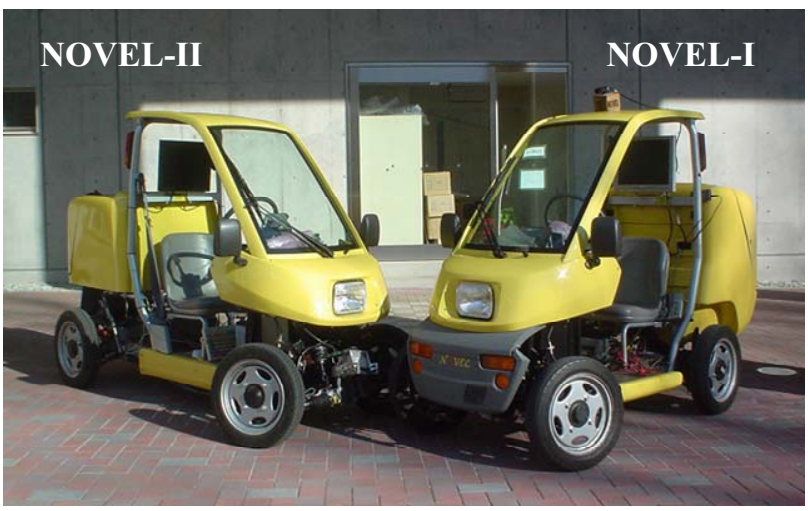

Fig. 1 Micro-scale electric vehicle NOVEL-I and NOVEL-II

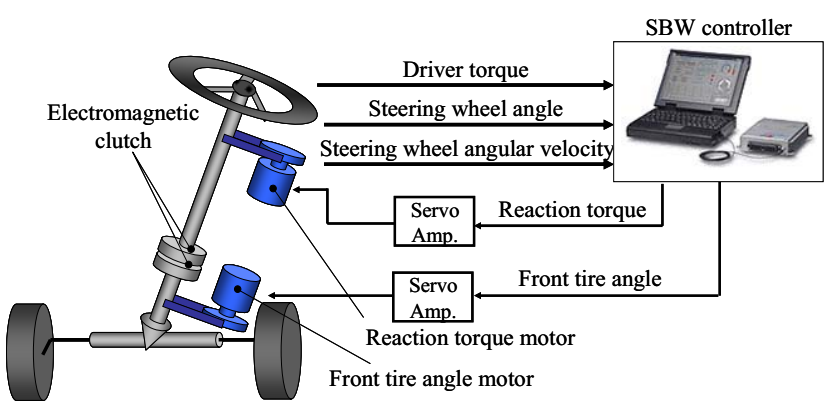

(a) Hardware configuration

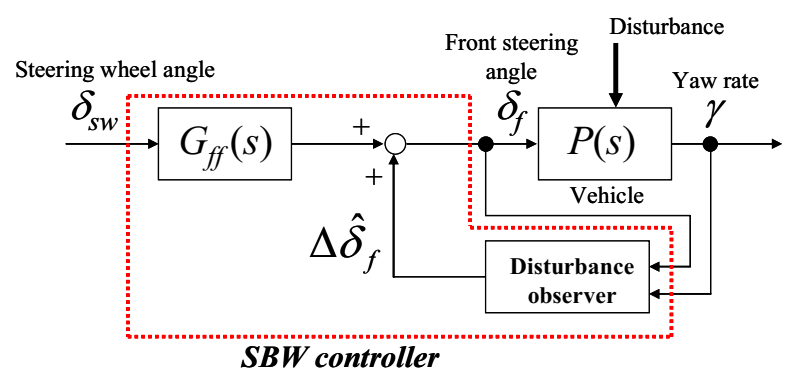

(b) Control system algorithm

Fig. 2 Configuration of steer-by-wire system

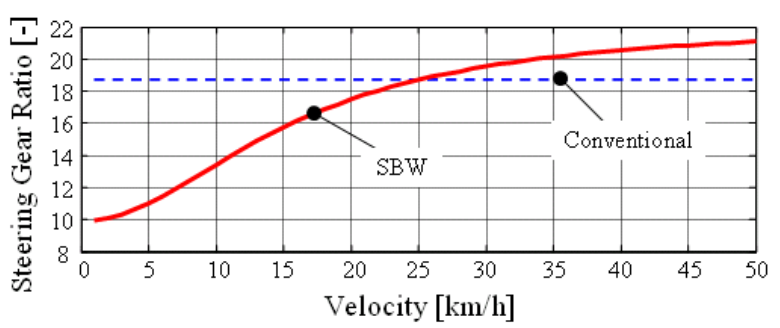

Fig. 3 Variable steering gear ratio depending on vehicle speed

The equivalent steering gear ratio of experimental vehicle by the proposed determination is indicated in Fig.3. From the experiments, Fig.4 shows the lissajous diagram of steering wheel angle and yaw rate during single lane change maneuver of NOVEL-II at velocity of $30 \mathrm{~km} / \mathrm{h}$. This figure shows that, the hysteresis of the 
yaw rate response to the steering angle input in the case of SBW control is smaller than that of vehicle without steering gear control function. This implies the improvement of vehicle handling by SBW application.

Next, the improvement of vehicle stability against disturbance was investigated in asymmetric braking on $\mu$-split road. Yaw rate feedback to front steering angle is applied to compensate external disturbance. The yaw rate feedback control algorithm is designed with the application of disturbance observer. The control input can be calculated by the following expression.

$$
\Delta \hat{\delta}_{f}=\frac{1}{T s+1}\left[P^{-1}(s) \gamma-\delta_{f}\right]
$$

where $\Delta \delta_{\mathrm{f}}$ denotes the additional steering angle front the controller, $T$ the time constant of the disturbance observer for noise rejection, $s$ the Laplacian operator and $P(\mathrm{~s})$ the simplified vehicle dynamics transfer function. The effectiveness of the feedback control system was verified by braking experiment on $\mu$-split road. From experimental results shown in Fig.5, the control system can effectively suppress the yaw rate deviation by controlling the front steering angle in real time.

\section{DIRECT YAW MOMENT CONTROL}

This section describes the design of direct yaw moment control system which utilizes the transverse driving torque distribution control of in-wheel-motors equipped at rear wheels of electric vehicle NOVEL-I. The direct yaw moment is used to control the vehicle position to be on the center of the lane, which is recognized by vision sensor, e.g. CCD camera. Such type of vehicle motion control can be applied to the driving assistance system, i.e. lane keeping assistance or lane departure prevention system.

\subsection{Control Algorithm[4]}

This paper applies the idea of the $2^{\text {nd }}$ order predictive model for calculating the desired yaw rate based on the information from CCD camera. As shown in Fig.6, From Taylor's $2^{\text {nd }}$ order expansion, the predicted lateral displacement at the distance in front of vehicle of $l_{s}$ can be expressed as follows:

$$
\begin{aligned}
& y_{c}(t+\Delta t)=y_{c}(t)+\Delta t \cdot \dot{y}_{c}(t)+\frac{(\Delta t)^{2}}{2} \cdot \ddot{y}_{c}(t) \\
& \Delta t=\frac{l_{s}}{V}
\end{aligned}
$$

As the objective of lane keeping control, the desired lateral displacement at preview point and the predicted lateral displacement must be equal.

$$
y_{c}(t+\Delta t)=y_{s}^{*}(t)
$$

Consequently, the required lateral acceleration for lane keeping can be calculated as follows:

$$
\begin{aligned}
\ddot{y}_{c}(t) & =\frac{2 V^{2}}{l_{s}^{2}}\left[y_{s}^{*}(t)-\left(y_{c}(t)+l_{s} \frac{\dot{y}_{c}(t)}{V}\right)\right] \\
& =-\frac{2 V^{2}}{l_{s}^{2}} y_{s r}(t)
\end{aligned}
$$

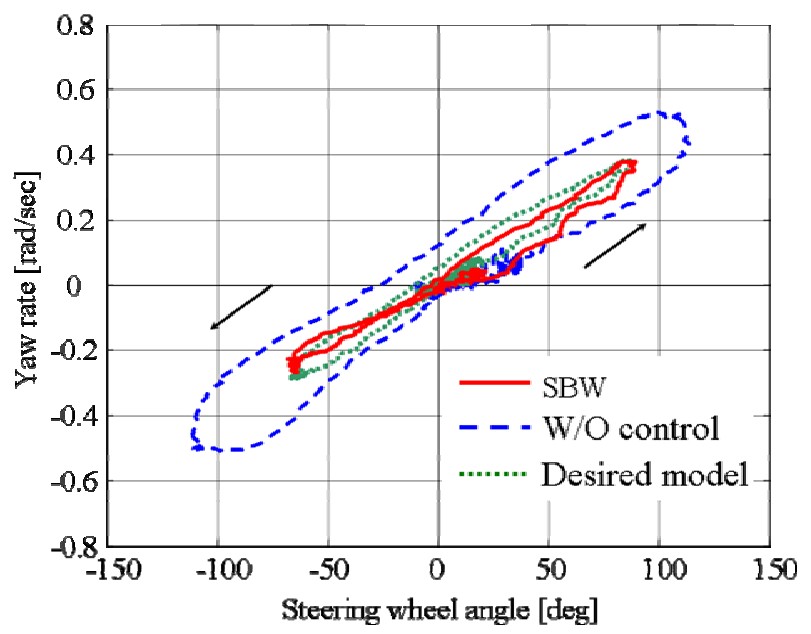

Fig. 4 Effect of steer-by-wire control in single lane-change test at speed of $30 \mathrm{~km} / \mathrm{h}$
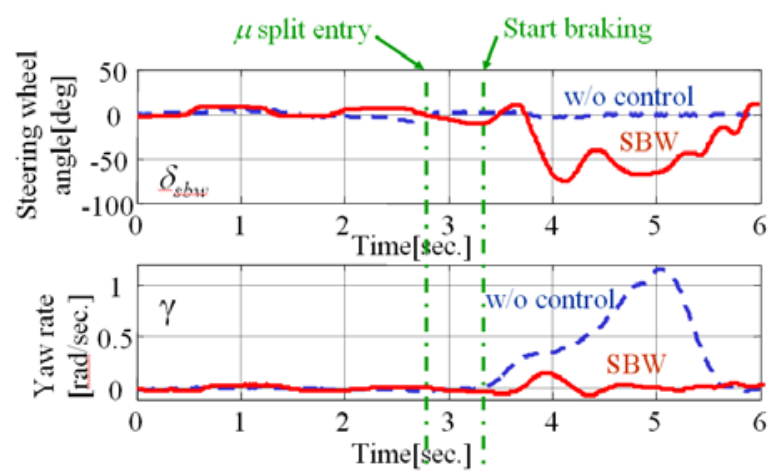

Fig. 5 Effect of steer-by-wire control during braking on $\mu$-split road

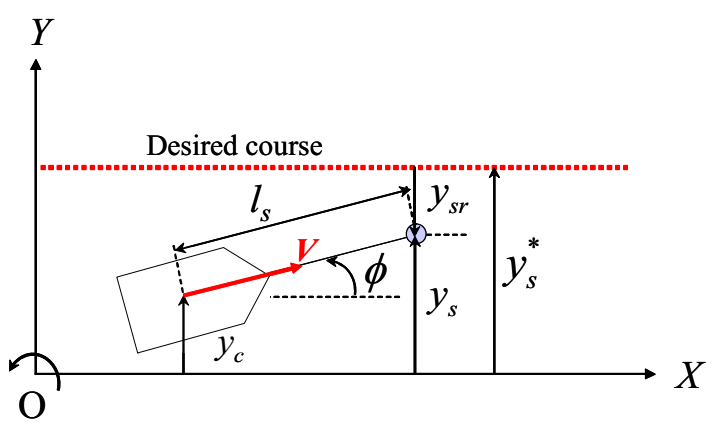

Fig. 6 Vehicle model in earth-fixed coordinate system

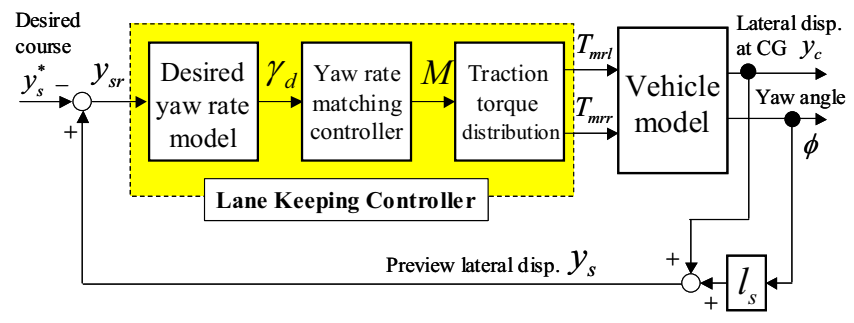

Fig. 7 Block diagram of lane keeping control system by DYC 
When the body side slip angle is negligible, the kinematics relationship between yaw rate and lateral acceleration can be expressed as follows:

$$
\ddot{y}_{c}(t)=V \gamma(t)
$$

By substituting Eq.(9) into Eq.(8), the desired yaw rate for lane keeping control can be obtained as follows:

$$
\gamma(t)=-\frac{2 V}{l_{s}^{2}} y_{s r}(t)
$$

In the case of curved lane tracking, the yaw rate according to the road curvature $\rho$ must be included.

Yaw rate at curved lane : $\gamma_{c}(t)=V \rho(t)$

Total yaw rate:

$$
\gamma_{d}(t)=\gamma(t)+\gamma_{c}(t)=-\frac{2 V}{l_{s}^{2}} y_{s r}(t)+V \rho(t)
$$

where, the road curvature can be estimated in real time from the vision information and vehicle lateral dynamics model with the application of Kalman filter.

To make the vehicle trace the desired yaw rate, the yaw moment controller calculates the direct yaw moment control input by using linear inverse dynamics. The transfer function from yaw moment input to yaw rate can be calculated as follows:

$$
\gamma(s)=\frac{a_{1} s+a_{o}}{s^{2}+b_{1} s+b_{o}} M(s)
$$

where, each coefficient can be expressed as follows:

$a_{1}=\frac{1}{I}, \quad a_{o}=\frac{2\left(C_{f}+C_{r}\right)}{m I V}$

$b_{1}=\frac{2\left(C_{f}+C_{r}\right)}{m V}+\frac{2\left(l_{f}^{2} C_{f}+l_{r}^{2} C_{r}\right)}{I V}, b_{o}=\frac{4 l^{2} C_{f} C_{r}}{m I V^{2}}-\frac{2\left(l_{f} C_{f}-l_{r} C_{r}\right)}{I}$

With the inverse transfer function of Eq.(13), the yaw moment input for tracing the desired yaw rate can be theoretically calculated as follows:

$$
M(s)=\frac{s^{2}+b_{1} s+b_{o}}{\left(a_{1} s+a_{o}\right)\left(\tau_{o} s+1\right)} \gamma_{d}(s)
$$

where, $\tau_{o}$ denotes the time constant of $1^{\text {st }}$ order lag element to make the transfer function of the controller become proper.

Practically, the yaw moment input is generated by the differential driving torque between left and right wheels. The driving torque of each motor can be calculated as follows:

Left wheel: $\quad T_{m r l}(t)=T_{s t}(t)-\frac{r_{w}}{d} M(t)$

$$
\text { Right wheel: } \quad T_{m r r}(t)=T_{s t}(t)+\frac{r_{w}}{d} M(t)
$$

where, $T_{s t}$ indicates the driving torque required for straight running. The block diagram of the proposed lane keeping control system is shown in Fig.7.

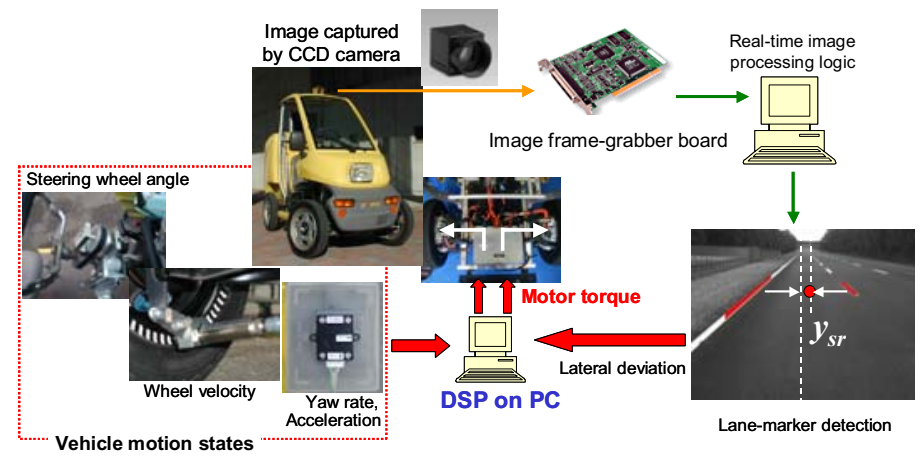

Fig. 8 System configuration in micro EV NOVEL-I

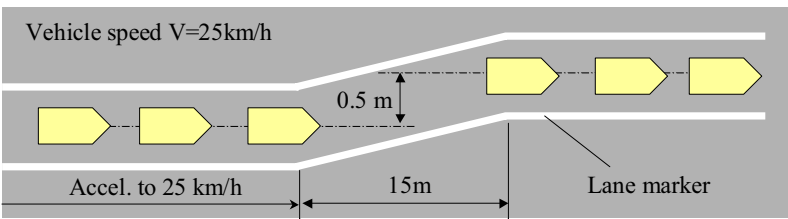

Fig. 9 Course layout for system validation (straight roadway tracking)
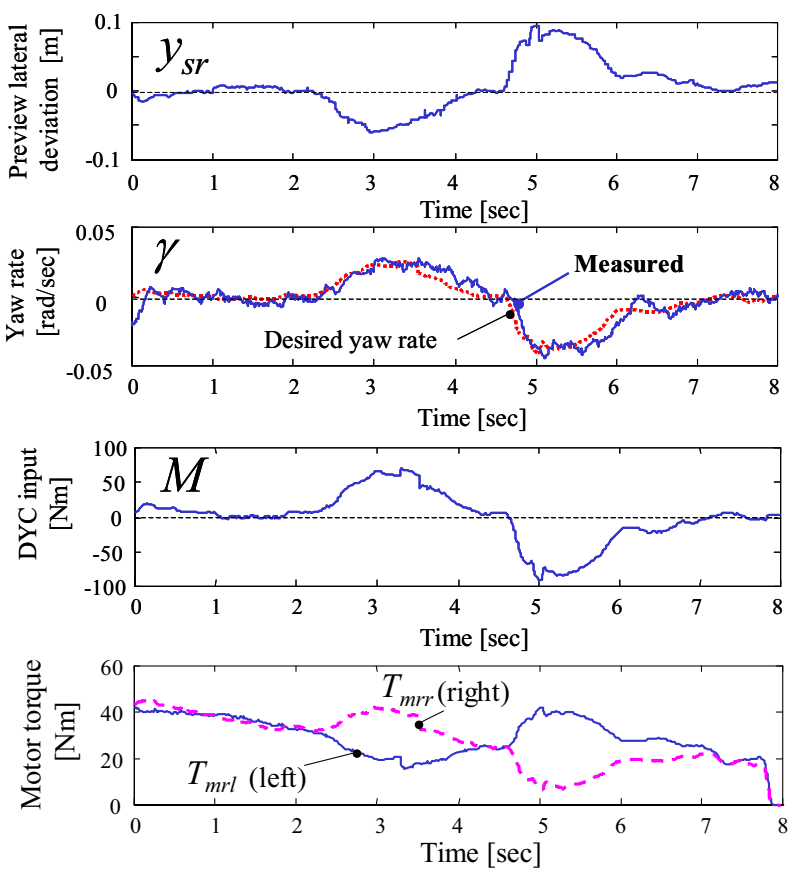

Fig. 10 Experimental results on straight lane $(\mathrm{V}=25 \mathrm{~km} / \mathrm{h})$

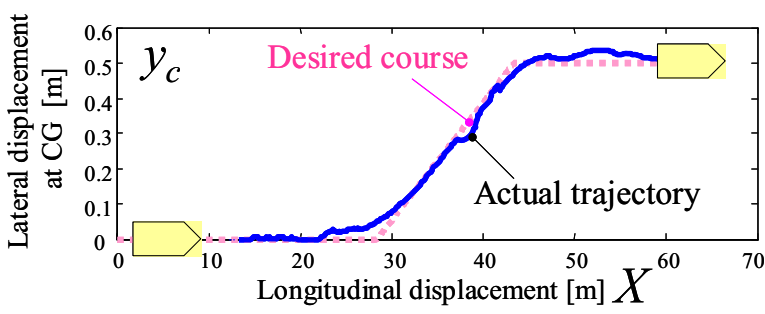

Fig. 11 Vehicle trajectory during lane keeping (experiment)

\subsection{Experiments on Straight Roadway}

The lane keeping control system configuration for experiments is indicated in Fig.8. First, the validity of the proposed lane keeping control system was proved 
on straight roadway with a small lane change course as shown in Fig.9. Fig.10 shows the experimental results in the case of straight lane. The vehicle ran straight and accelerated to velocity of $25 \mathrm{~km} / \mathrm{h}$ and passed through the course shifting region without driver's corrective steering. Fig.10 shows the time history of preview lateral deviation measured from the CCD camera, vehicle yaw rate, front steering angle, DYC input and in-wheel-motor torques. The measured yaw rate shows good consistency with the desired yaw rate, which means the yaw moment controller by yaw rate matching control method is effective. The in-wheel-motor torques shows that the individual wheel torque control was actually realized. Fig. 11 shows the trajectory of vehicle centre of gravity, which shows that the vehicle actually traced the set course by the proposed DYC.

\subsection{Experiments on Curved Roadway}

Next, the validity of the proposed lane keeping control system was proved on curved roadway with constant radius of curvature of $100 \mathrm{~m}$. In the experiment, the effectiveness of the curvature estimator and the DYC system were verified. The driver ran the vehicle at velocity of $35 \mathrm{~km} / \mathrm{h}$ and entered the curved lane which has constant turning radius of $100 \mathrm{~m}$. During tracing the curved roadway, the driver did not hold the steering wheel. The experimental data is shown in Fig.12.

Fig.12 shows the time responses of the preview lateral deviation, the vehicle yaw rate, the estimated road curvature, the DYC control input, the in-wheel-motor torques. The lateral deviation was suppressed under $50 \mathrm{~cm}$, which was quite satisfactory. The vehicle yaw rate in steady state traced the reference value well. The estimated road curvature was satisfactorily consistent with the reference road curvature.

\subsection{Effect of DYC on Driver-Vehicle System}

This subsection investigates the effectiveness of the proposed DYC on driver-vehicle system. The desired course is set for the experiment as shown in Fig. 13. The driver drove the vehicle to run straight and accelerate to velocity of $25 \mathrm{~km} / \mathrm{h}$ and after that the driver steered the vehicle in order to trace the determined course. In the experiment, the driver was told to trace the center of the lane as possible. The time history of the experiment conducted by a driver is shown in Fig. 14.

Fig.14 shows the experimental results in the case of without DYC assist and the case of DYC assist. From the figure, with the DYC assist, the vehicle lateral deviation was effectively reduced, which means the improvement of lane keeping control performance. From the response of yaw rate, DYC improved the yaw rate responsiveness when the vehicle tried to change the direction by initiating the yaw rate before the driver began to steer the vehicle, and also improved the stability of yaw rate when the vehicle returned to straight driving. From the time history of steering wheel angle in Fig.14, it was found that the steering operation of driver was effectively reduced as DYC provided yaw moment for lane keeping. This means that the driver steering physical workload was reduced, and such control effect leads to comfort drive.
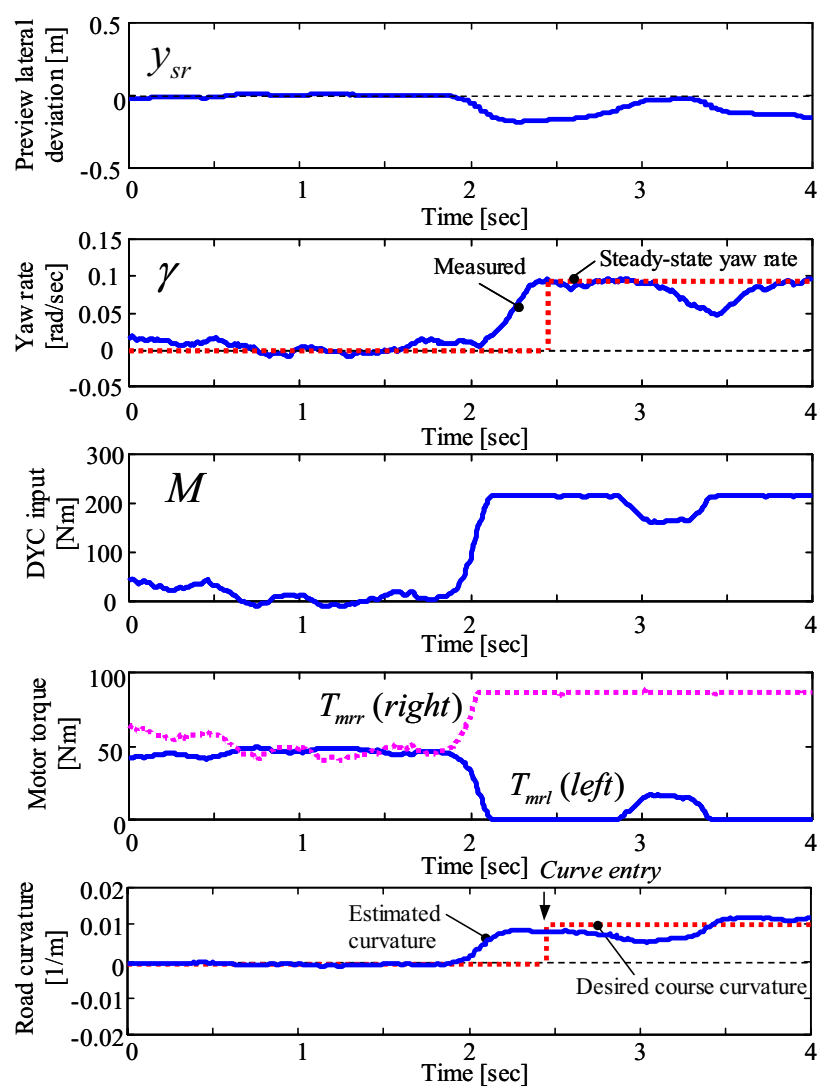

Fig. 12 Experimental results on 100R curved lane $(\mathrm{V}=35 \mathrm{~km} / \mathrm{h})$

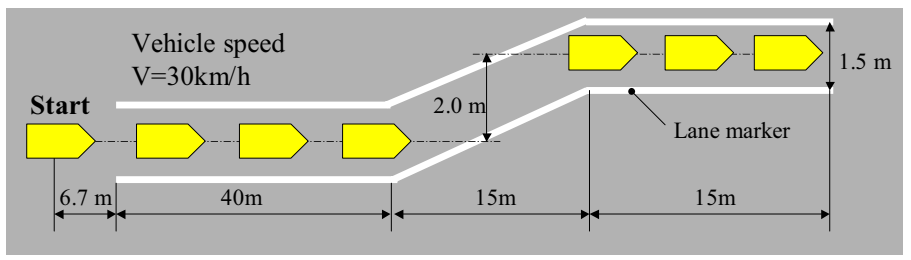

Fig. 13 Course layout for experiments on straight lane (section 4.4)

Next, the effectiveness of DYC is investigated on curved lane. The vehicle ran at a constant velocity of 30 $\mathrm{km} / \mathrm{h}$ on straight lane at distance of $40 \mathrm{~m}$ and then entered the curve lane, which has curve radius of $40 \mathrm{~m}$.

Fig. 15 shows the experimental results in the case of without DYC assist and the case of DYC assist. From the figure, both cases show the same time history of lateral deviation and vehicle yaw rate. This means that the vehicle almost ran at the same trajectory even DYC acted on the vehicle for driving assistance. The significant difference of time history between two cases appeared on the steering wheel angle response. With DYC assist, the yaw moment for tracing the curved lane was generated actively, and reduced the driver steering 
wheel angle. This shows the same effect as the case of straight lane. The proposed DYC effectively reduces the steering physical workload, and such control effect leads to comfort drive.
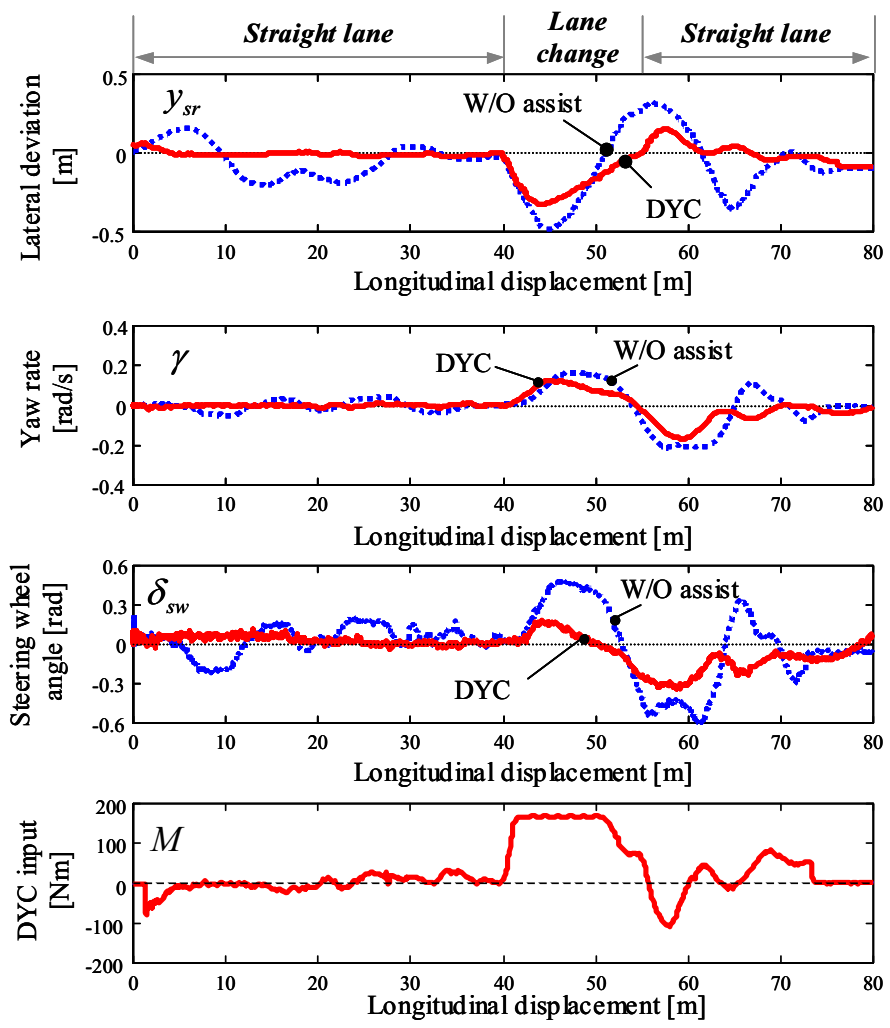

Fig. 14 Experimental results on straight lane

(driver-vehicle system)
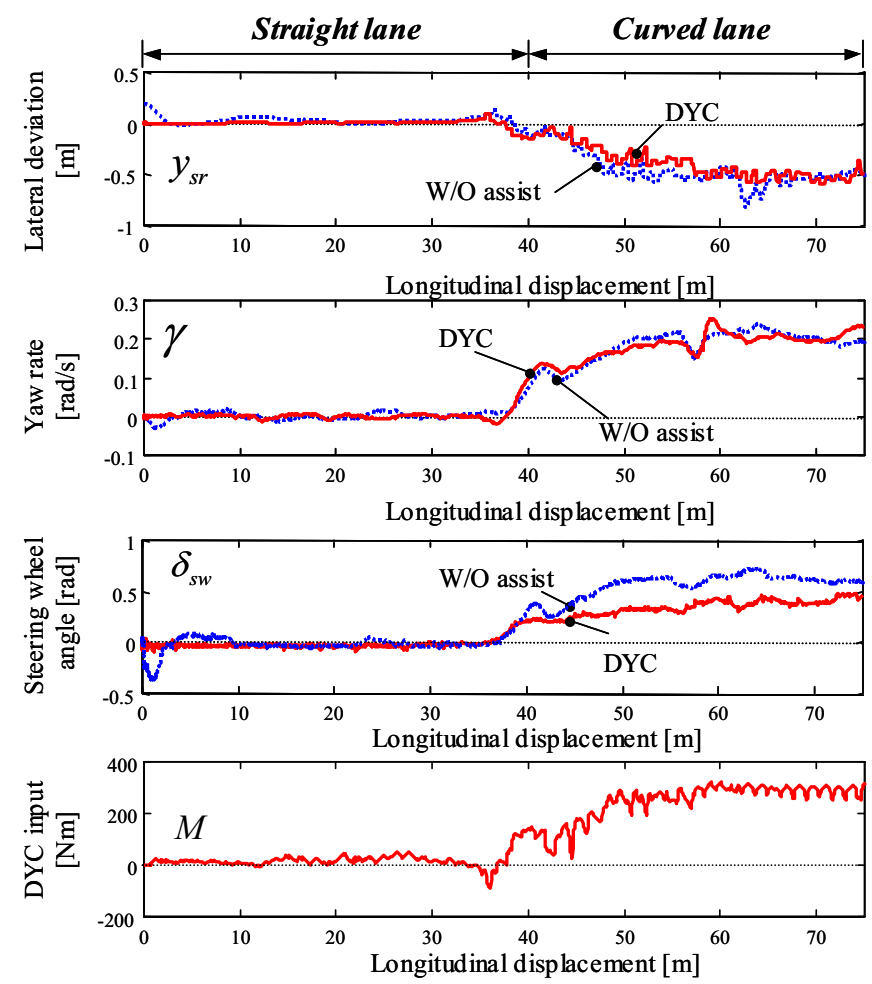

Fig. 15 Experimental results on curved lane (driver-vehicle system)

\section{CONCLUDING REMARKS}

This article presented the motion control issues and applications of using electric motors in actual micro-scale electric vehicle. Two types of control systems were described in the article. First, the active front steering control with servomotor was demonstrated. The effectiveness of the system on handling and stability improvement was verified. Next, the direct yaw moment control with in-wheel-motor torque distribution was demonstrated for lane keeping task. The lane keeping control system design was shown and the validation of the system was conducted. Finally, the effect on steering workload reduction of the proposed DYC was examined on driver-vehicle system.

This is the way to ensure that the active safety of the small-size electric vehicle can be secured by various types of controllers. The next step of the study is to adapt the controller to the driver intention or driving characteristics. In future, large-scale driving database storage is required to conduct this issue.

\section{REFERENCES}

[1] Raksincharoensak, P., Shino, M. and Nagai, M., "Enhancing Active Safety of Small-Scale Electric Vehicle "NOVEL" by Utilizing In-Wheel-Motors", Proceedings of the $20^{\text {th }}$ International Electric Vehicle Symposium and Exposition, California. EDTA, 2003.

[2] Shino, M., Raksincharoensak, P., Kamata, M. and Nagai, M., "Side Slip Control of Small-Scale Electric Vehicle by DYC", Vehicle System Dynamics Supplement Vol.41, p.487-496. Taylor \& Francis, 2004.

[3] Shino, M., Watanabe, S. , Raksincharoensak, P. and Nagai, M., Vehicle Handling and Stability Control of Micro-Scale Electric Vehicle Utilizing Steer-by-Wire System, Proceedings of AVEC'04 Symposium, pp.797-802, 2004.

[4] Raksincharoensak, P., Nagai, M. and Shino, M., "Lane Keeping Control Strategy with Direct Yaw Moment Control Input by Considering Dynamics of Electric Vehicle", Vehicle System Dynamics Supplement Vol.44, Taylor \& Francis, 2006. 


\section{APPENDIX}

\section{NOTATIONS}

$m \quad$ Vehicle mass

$M \quad$ Yaw moment control input

I Yaw moment of inertia

$l_{f} \quad$ Distance from centre of gravity to front axle

$l_{r} \quad$ Distance from centre of gravity to rear axle

$C_{f} \quad$ Front tyre cornering stiffness (1 wheel)

$C_{r} \quad$ Rear tyre cornering stiffness (1 wheel)

$d \quad$ Vehicle tread

$r_{w} \quad$ Tyre effective radius

$V \quad$ Vehicle speed

$l_{\mathrm{s}} \quad$ Preview distance of CCD camera

$y_{c} \quad$ Lateral displacement at centre of gravity

$y_{s r} \quad$ Lateral deviation at camera preview point

$\beta \quad$ Vehicle side slip angle

$\gamma \quad$ Vehicle yaw rate

$\rho \quad$ Road curvature

$F_{x r l}\left(F_{x r r}\right)$ Longitudinal forces of left (right) tyre

$T_{m r l}\left(T_{m r r}\right)$ Longitudinal forces of left (right) tyre

\section{BIOGRAPHIES}

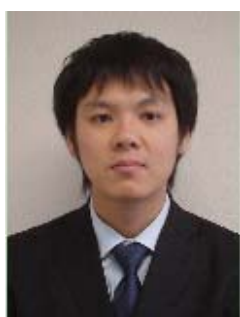

Dr.Pongsathorn Raksincharoensak, Department of Mechanical Systems Engineering, Tokyo University of Agriculture and Technology, received Master degree and Ph.D. degree in mechanical engineering from Tokyo University of Agriculture and Technology in 2002 and 2005. Currently, he is working as an associate professor in department of mechanical systems engineering of TUAT. His research interests are automotive safety-related devices especially dynamics and control application to vehicle handling and stability enhancement, driving assistance system for ITS applications, hybrid electric vehicle control for improving fuel economy, motion control of electric vehicle and etc.

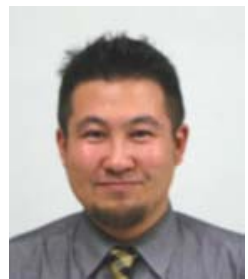

Motoki Shino, Department of Engineering Synthesis, The University of Tokyo, received Ph.D. degree in mechanical engineering from Tokyo University of Agriculture and Technology in 2002. His research interests are dynamics and control of vehicle to enhance handling and stability of vehicle. Currently, he is working as an assistant professor in department of engineering synthesis, the University of Tokyo. He is working on research issues relating to the analysis of driving behaviors of aged drivers, and the design of innovative vehicle operating systems.

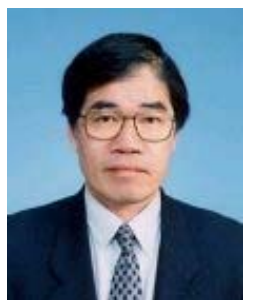

Prof. Masao Nagai, Department of Mechanical Systems Engineering, Tokyo University of Agriculture and Technology, received $\mathrm{Ph} . \mathrm{D}$. degree in mechanical engineering from the University of Tokyo in 1977. Since 1978, he has been with T.U.Braunschweig as visiting researcher under Prof. Manfred Mitschke for 2 years. His main research interests are dynamics and control applications for automobiles and railway vehicles. He is author of books on Dynamics and Control of Vehicle Systems (JSME textbook series, 1999). Prof. Nagai is recipient of Japan Society of Mechanical Engineering (JSME) Research Achievement Awards in 2001 and numerous prizes from JSAE for excellent papers. 